\title{
Multiple behaviour change intervention and outcomes in recently diagnosed type 2 diabetes: the ADDITION-Plus randomised controlled trial
}

\author{
Simon J. Griffin • Rebecca K. Simmons • A. Toby Prevost • Kate M. Williams • \\ Wendy Hardeman • Stephen Sutton • Søren Brage • Ulf Ekelund • \\ Richard A. Parker • Nicholas J. Wareham • Ann Louise Kinmonth • \\ on behalf of the ADDITION-Plus study team
}

Received: 4 December 2013 / Accepted: 4 March 2014 / Published online: 24 April 2014

(C) The Author(s) 2014. This article is published with open access at Springerlink.com

\begin{abstract}
Aims/hypothesis The aim of this study was to assess whether or not a theory-based behaviour change intervention delivered by trained and quality-assured lifestyle facilitators can achieve and maintain improvements in physical activity, dietary change, medication adherence and smoking cessation in people with recently diagnosed type 2 diabetes.

Methods An explanatory randomised controlled trial was conducted in 34 general practices in Eastern England (AngloDanish-Dutch Study of Intensive Treatment in People with Screen Detected Diabetes in Primary Care-Plus [ADDITIONPlus]). In all, 478 patients meeting eligibility criteria (age 40 to 69 years with recently diagnosed screen or clinically detected diabetes) were individually randomised to receive either
\end{abstract}

Electronic supplementary material The online version of this article (doi:10.1007/s00125-014-3236-6) contains peer-reviewed but unedited supplementary material, which is available to authorised users.

S. J. Griffin $(\varangle) \cdot$ R. K. Simmons $\cdot$ S. Brage $\cdot$ U. Ekelund

N. J. Wareham

MRC Epidemiology Unit, University of Cambridge School of

Clinical Medicine, Cambridge, UK

e-mail: simon.griffin@mrc-epid.cam.ac.uk

S. J. Griffin • K. M. Williams • W. Hardeman • S. Sutton •

R. A. Parker $\cdot$ A. L. Kinmonth

The Primary Care Unit, Department of Public Health and Primary

Care, University of Cambridge, Robinson Way,

Cambridge CB2 0SR, UK

A. T. Prevost

Department of Primary Care and Public Health Sciences,

King's College London, London, UK

U. Ekelund

Department of Sports Medicine, Norwegian School of Sport

Sciences, Oslo, Norway intensive treatment $(n=239)$ or intensive treatment plus a theory-based behaviour change intervention led by a facilitator external to the general practice team $(n=239)$. Randomisation was central and independent using a partial minimisation procedure to balance stratifiers between treatment arms. Facilitators taught patients skills to facilitate change in and maintenance of key health behaviours, including goal setting, selfmonitoring and building habits. Primary outcomes included physical activity energy expenditure (individually calibrated heart rate monitoring and movement sensing), change in objectively measured fruit and vegetable intake (plasma vitamin $\mathrm{C}$ ), medication adherence (plasma drug levels) and smoking status (plasma cotinine levels) at 1 year. Measurements, data entry and laboratory analysis were conducted with staff unaware of participants' study group allocation. Results Of 475 participants still alive, 444 (93\%; intervention group $95 \%$, comparison group 92\%) attended 1-year followup. There were no significant differences between groups in physical activity (difference: $+1.50 \mathrm{~kJ} \mathrm{~kg}^{-1}$ day $^{-1} ; 95 \% \mathrm{CI}$ $-1.74,4.74$ ), plasma vitamin $\mathrm{C}$ (difference: $-3.84 \mu \mathrm{mol} / \mathrm{l}$; $95 \%$ CI $-8.07,0.38$ ), smoking (OR 1.37 ; 95\% CI 0.77 , 2.43) and plasma drug levels (difference in metformin levels: $-119.5 \mu \mathrm{mol} / 1 ; 95 \%$ CI $-335.0,95.9)$. Cardiovascular risk factors and self-reported behaviour improved in both groups with no significant differences between groups.

Conclusions/interpretation For patients with recently diagnosed type 2 diabetes receiving intensive treatment in UK primary care, a facilitator-led individually tailored behaviour change intervention did not improve objectively measured health behaviours or cardiovascular risk factors over 1 year. Trial registration ISRCTN99175498

Funding The trial is supported by the Medical Research Council, the Wellcome Trust, National Health Service R\&D support funding (including the Primary Care Research and 
Diabetes Research Networks) and National Institute of Health Research under its Programme Grants for Applied Research scheme. The Primary Care Unit is supported by NIHR Research funds. Bio-Rad provided equipment for $\mathrm{HbA}_{1 \mathrm{c}}$ testing during the screening phase.

Keywords ADDITION-Plus · Diabetes · General practice · Health behaviour $\cdot$ Randomised trial

$\begin{array}{ll}\begin{array}{ll}\text { Abbreviations } \\ \text { ADDITION }\end{array} & \begin{array}{l}\text { Anglo-Danish-Dutch Study of Intensive } \\ \text { Treatment in People with Screen Detected } \\ \text { Diabetes in Primary Care } \\ \text { Consultation and Relational Empathy }\end{array} \\ \text { CARE } & \text { Cardiovascular disease } \\ \text { CVD } & \text { Diabetes Education and Self-Management } \\ \text { DESMOND } & \text { for Ongoing and Newly Diagnosed } \\ & \text { Diabetes } \\ \text { Early ACTID } & \text { Early Activity in Diabetes } \\ \text { EQ-5D } & \text { EuroQol 5-D } \\ \text { Look-AHEAD } & \text { Action for Health Diabetes } \\ \text { MARS } & \text { Medication Adherence Report Schedule } \\ \text { PAEE } & \text { Physical activity energy expenditure } \\ \text { SF-36 } & \text { Short-Form 36 } \\ \text { TPB } & \text { Theory of planned behaviour } \\ \text { UKPDS } & \text { UK Prospective Diabetes Study }\end{array}$

\section{Introduction}

Well-organised care, including regular recall and review of patients, prompting of doctors, feedback on goal attainment, and continuing medical education and guidelines, are associated with reductions in risk factors among people with type 2 diabetes $[1,2]$. In addition, intensive pharmacological treatment of risk factors can reduce cardiovascular morbidity and mortality [3, 4]. However, these benefits depend on people with diabetes taking their medication as prescribed, eating a healthy diet, being physically active on a regular basis and avoiding smoking.

There is some evidence that patient education, training patients in self-management and using interventions incorporating well-specified behaviour change techniques can be effective, at least in the short term [5]. However, only a minority of people with type 2 diabetes in the UK have attended a structured education programme to assist with behaviour change, and where attendance has occurred the effectiveness of the programme in practice is largely uncertain [6].

The Early Activity In Diabetes (Early ACTID: ISRCT N92162869) and Action for Health Diabetes (Look-AHEAD: NCT00017953) trials have demonstrated beneficial effects on cardiovascular risk factors of adding intensive lifestyle interventions to the primary care of diabetes patients [7, 8]. These studies were selective in their behavioural focus. Many previous studies have been conducted in research clinics or specialist settings and have not clearly characterised the comparison condition. Behavioural interventions and their hypothesised mechanisms of action are rarely clearly specified and the delivery of the intervention is not often assessed. Most trials have relied on self-reported measures of behaviour, which are imprecise and subject to recall bias, and do not assess adherence to medication. This limits evaluation of the effects of behavioural interventions independent of organisational and pharmacological components, and cannot inform subsequent integration of the most effective components into routine practice.

We aimed to address these uncertainties in the AngloDanish-Dutch Study of Intensive Treatment in People with Screen Detected Diabetes in Primary Care-Plus (ADDITIONPlus) trial by evaluating the effect of a theory-based behaviour change intervention delivered by trained and quality-assured lifestyle facilitators, external to the primary care team, on objectively measured health behaviours (physical activity, diet change, medication adherence and smoking) among people with recently diagnosed type 2 diabetes receiving intensive general practice care [9].

\section{Methods}

Study design

The study design and rationale have been reported previously (2002-2007) [9]. Patients were recruited from 34 general practices in the East of England. The majority of practices $(n=26)$ were participating in the intensive treatment arm of the ADDITION-Cambridge trial of treatment of people with diabetes detected by screening (ISRCTN86769081) [10]. A further eight practices were recruited to increase the participation of recently clinically diagnosed patients (Fig. 1). Eligibility criteria were initially assessed by general practice staff and included age 40-69 years with type 2 diabetes following screening in the ADDITION study or clinical diagnosis during the previous 3 years. Exclusion criteria included patients who had a psychotic illness or an illness with a likely prognosis of $<1$ year and women who were pregnant or lactating. Of 425 eligible screendetected patients and 684 patients clinically diagnosed within the previous 3 years, 239 from each group agreed to be individually randomised to receive intensive treatment alone (comparison group) or in conjunction with a facilitator-led behaviour change intervention (intervention group).

Randomisation

Randomisation was central using a partial minimisation procedure to balance stratifiers (age, sex and general practice; and 
Fig. 1 Study design and participant flow in the ADDITION-Plus trial

\begin{tabular}{|c|}
\hline $\mathrm{E}$ \\
$\mathrm{N}$ \\
$\mathrm{R}$ \\
$\mathrm{O}$ \\
$\mathrm{L}$ \\
$\mathrm{M}$ \\
$\mathrm{E}$ \\
$\mathrm{N}$ \\
$\mathrm{T}$ \\
\hline
\end{tabular}

\begin{tabular}{|l|}
\hline $\mathrm{A}$ \\
$\mathrm{L}$ \\
$\mathrm{L}$ \\
$\mathrm{O}$ \\
$\mathrm{C}$ \\
$\mathrm{A}$ \\
$\mathrm{T}$ \\
$\mathrm{I}$ \\
$\mathrm{O}$ \\
$\mathrm{N}$ \\
\hline
\end{tabular}

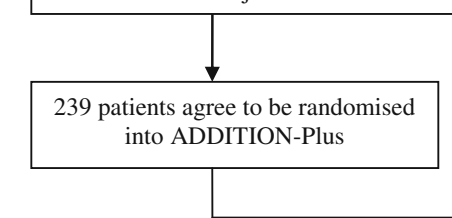

239 patients agree to be randomised into ADDITION-Plus

26 practices nested within the intensive treatment arm of the ADDITION-Cambridge trial, with $n=425$ screen-detected type 2 diabetes patients invited to join ADDITION-Plus

27 practices (19 ADDITION-Cambridge, 8 nonADDITION practices), with $n=684$ type 2 diabetes patients diagnosed within the previous 3 years invited to join ADDITION-Plus

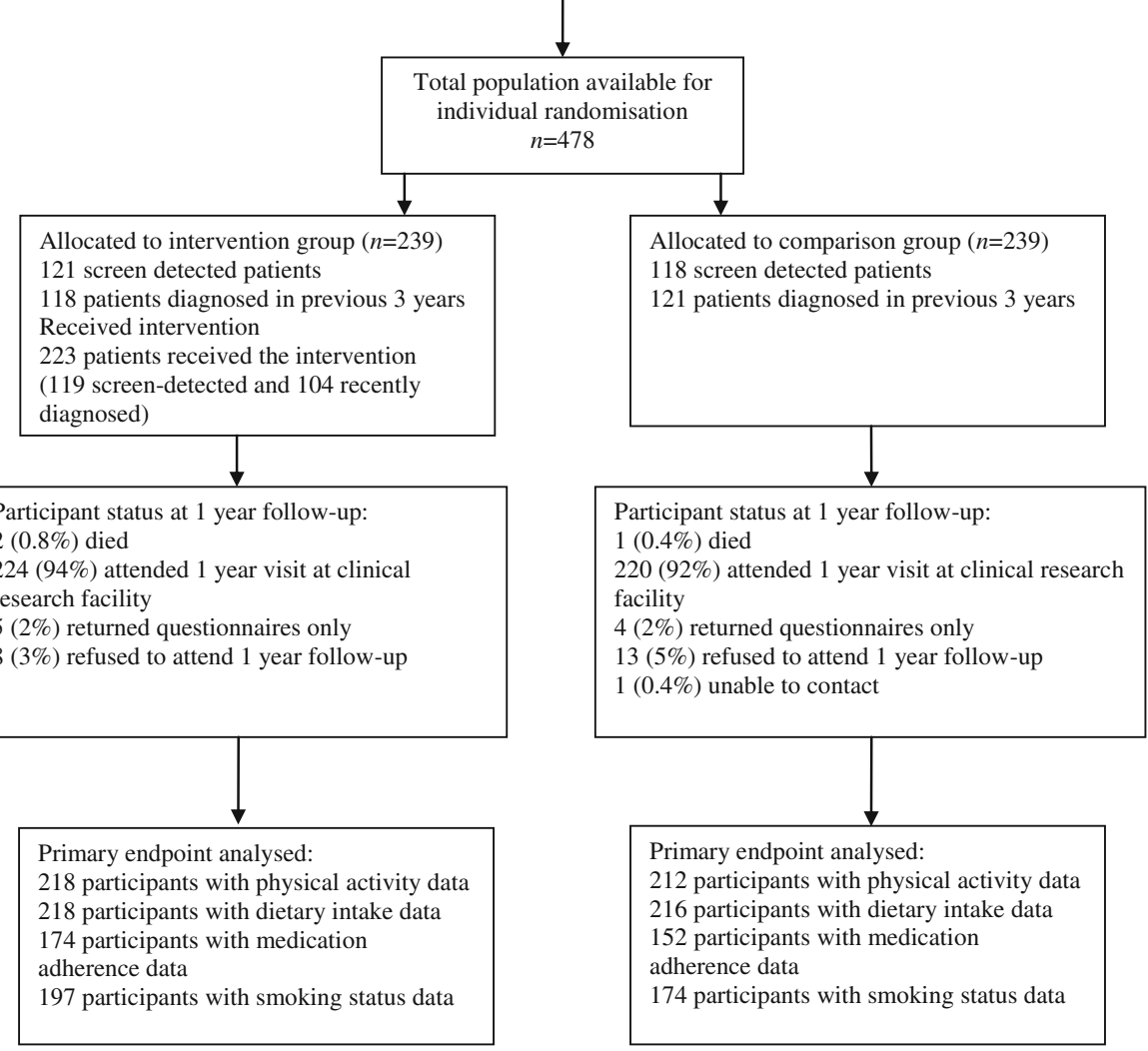

within screen-detected and clinically diagnosed subgroups: BMI, self-reported smoking and medication adherence [11]) between the arms [9]. Randomisation was undertaken independently of study coordination or knowledge of or contact with participants or their data, other than the stratifiers. Ethical approval was obtained from the Eastern MultiCentre Research Ethics Committee (reference no. 02/5/54). All participants provided written informed consent.

Intervention

Comparison group: intensive treatment A number of features were added to routine multidisciplinary primary care of diabetes to achieve intensive treatment in both trial groups as previously described [9, 10] (see Electronic Supplementary Material [ESM] for further details).

Intervention group: intensive treatment plus facilitator-led behaviour change intervention Participants in the intervention group received intensive treatment (described above) plus a facilitator-led, individually tailored behaviour change intervention, based on psychological theory and evidence. Full details of the intervention have been described previously (see ESM) [9]. In brief, the intervention was delivered by three female trained lifestyle facilitators, who were not part of the general practice team. The facilitators used detailed protocols to guide each contact with the participant and received ongoing supervision and feedback from a clinical psychologist. The behaviours targeted in the intervention were physical activity, dietary intake, medication adherence and smoking cessation. Facilitators taught patients a range of selfregulatory skills to achieve behaviour change and maintenance over time, supported by a manual describing the skills. The intervention was delivered over 1 year at the participants' surgeries and included a $1 \mathrm{~h}$ introductory meeting followed by six 30 min meetings and four brief phone calls. 
Measurements and endpoints

Baseline and 1 year measurements were undertaken at outpatient clinical research facilities by trained staff following standard operating procedures. Double data entry of anthropometric and questionnaire measures was undertaken by independent agencies. Measurements, data entry and laboratory analysis were conducted with staff unaware of participants' study group allocation.

\section{Primary outcomes}

Objectively measured health behaviours Physical activity was assessed at 1 year using a combined heart rate and movement sensor (Actiheart, CamNtech, Cambridge, UK), which participants were advised to wear continuously for at least 4 days [12]. A graded treadmill walk test was used to individually calibrate heart rate [13] and to estimate cardiorespiratory fitness by extrapolation of the heart rate/oxygen consumption relationship to age-predicted maximal heart rate [14]. Timeseries data were pre-processed [15] and summarised into physical activity energy expenditure (PAEE, $\mathrm{kJ} \mathrm{kg}^{-1}$ day $^{-1}$ ) [16]. Data from participants without a treadmill test $(n=79)$ were processed using an adjusted group calibration equation based on treadmill tests.

Intake of fruit and vegetables was assessed at baseline and 1 year by measurement of plasma vitamin $\mathrm{C}$ levels using a Fluoroskan Ascent FL fluorometer (Fisher Scientific UK, Loughborough, UK) [17]. Smoking status was assessed at 1 year by analysis of plasma cotinine levels using an Immulite Nicotine metabolite solid phase competitive chemiluminescent immunoassay (Siemens Healthcare Diagnostics, Llanberis, UK) and self-report. Self-reported non-smokers with cotinine levels exceeding $\geq 15 \mathrm{ng} / \mathrm{ml}(n=12)$ were re-classified as smokers [18]. Participants were advised to take their medication as usual on the day of testing. Medication adherence was assessed at 1 year follow-up by measurement of plasma concentrations of the three drugs that provided maximum participant coverage (metformin, simvastatin and atorvastatin) using liquid-chromatography-mass-spectrometry after protein precipitation extraction [9].

\section{Secondary outcomes}

Self-reported health behaviours Physical activity, dietary intake and adherence to hypoglycaemic and other medication were assessed using the previously validated EPIC-Norfolk Physical Activity Questionnaire [19], Food Frequency Questionnaire [20] and the Medication Adherence Report Schedule (MARS) [11], respectively. At 1 year, participants were asked whether or not they had made changes to their physical activity and dietary behaviours in the preceding 12 months.
Modelled risk of cardiovascular disease This was calculated for individuals without a prior cardiovascular disease (CVD) event using the UK Prospective Diabetes Study (UKPDS) model [21].

Biochemical and clinical measures Biochemical measures were assessed using standard assays (ESM) [9]. Detailed measurement protocols for blood pressure, height, weight, waist circumference and body fat percentage have been described previously [9]. Angina was assessed using the Rose angina questionnaire [22]. Neuropathy was evaluated using an adapted version of the Michigan Neuropathy Screening Instrument [23].

Functional status, healthy utility, anxiety, well-being, quality of life, treatment satisfaction and relational empathy The following generic and disease-specific instruments were used: Short-Form 36 (SF-36) [24], EuroQol (EQ-5D) [25], the short form of the state scale of the Spielberger State-Trait Anxiety Inventory [26], diabetes Well-Being Questionnaire [27], Audit of Diabetes Dependent Quality of Life [27] and Diabetes Treatment Satisfaction Questionnaire [27]. All patients completed the Consultation and Relational Empathy (CARE) measure in relation to the general practitioner and practice nurse [28], and a question concerning satisfaction with diabetes services. In addition, participants in the intervention group completed the CARE measure in relation to the lifestyle facilitator.

Beliefs about behaviour change, illness perceptions and habit A questionnaire developed according to the theory of planned behaviour (TPB) model [29, 30] assessed selected cognitions about becoming more physically active, eating a lower-fat diet, taking medication and smoking cessation: intention, perceived behavioural control and behavioural beliefs. Full details are reported elsewhere [9]. Participants also completed the consequences and treatment control subscales (11 items) of the Illness Perception Questionnaire-Revised $[31,32]$ and a nine-item closed-response questionnaire covering basic knowledge of diabetes and its management [33]. At 1 year, participants were asked to write down the most important change they had made in their physical activity and dietary intake [34]. Cronbach's alpha for TPB measures and the Illness Perception Questionnaire measures demonstrated high internal consistency. However, internal consistency was low $(0.55)$ for diabetes treatment control and perceived behavioural control in relation to stopping smoking.

Statistical analysis

We planned to recruit 500 participants. Follow-up of 400 individuals would provide $80 \%$ power at the $5 \%$ level of significance to detect between-arm differences of 
$0.017 \mathrm{~kJ} \mathrm{~kg}^{-1} \mathrm{~min}^{-1}$ in PAEE (anticipating a mean [SD] of $0.078[0.058] \mathrm{kJ} \mathrm{kg}^{-1} \mathrm{~min}^{-1}$ ) [35], $4.0 \mu \mathrm{mol} / 1$ in change from baseline in plasma vitamin $\mathrm{C}$ adjusted further for baseline (based on a mean [SD] of 53 [19] $\mu \mathrm{mol} / 1$ and test-retest correlation of 0.67 [36]) and $9.5 \%$ in smoking prevalence (control prevalence 17.9\%).

The effectiveness of the intervention on primary outcomes was assessed using between-arm intention-to-treat analysis, using linear and logistic regression for continuous and binary outcomes, respectively. Continuous outcomes adjusted for baseline where measured. Participants with missing baseline values were retained in the analysis by using the missing indicator method [37]. Effect sizes were reported with $95 \%$ CIs. Statistical significance was set at $p<0.05$. Continuous outcomes indicating non-normality or skewness (simvastatin, atorvastatin, triacylglycerol, alanine aminotransferase and UKPDS risk score) were natural log-transformed before analysis and their CIs were shown on the log-scale. Where non-normality could not be resolved by log-transformation, 95\% CIs were estimated using semi-parametric bias corrected and accelerated bootstrap regression with 1,999 resamples. We conducted a per protocol analysis of each primary outcome, in which the per protocol population comprised comparison-arm participants and those intervention-arm participants who attended the introductory and initial three coreintervention sessions. Sensitivity analysis was undertaken to assess the impact of excluding participants with missing primary outcome data. Rubin's multiple imputation method with 30 multiple imputation datasets [38] was used together with two contrasting scenarios for the intervention effect in excluded participants. The first optimistically assumed that excluded participants achieved the same full intervention effect as observed in participants with the outcome data, and the second pessimistically assumed that no intervention effect was achieved. Subgroup analyses were confined to the comparison of intervention effects on primary outcomes by mode of diagnosis (screen-detected or recently diagnosed).

\section{Results}

Figure 1 shows the trial profile. Participating practices were largely comparable with the average English practice in terms of list size, diabetes prevalence and general practice/nurse whole-time equivalents (data taken from the National Primary Care Database; www.population-health.manchester.ac.uk/ primarycare/npcrdc-archive/archive/ProjectDetail.cfm/ID/10. htm). However, the median (interquartile range) Index of Multiple Deprivation score for ADDITION-Plus practices (11.7 [6.6-15.5]) suggested that they served less deprived communities than the average English practice (21.2 [12.236.1]) (data taken from the UK National Primary Care Database). Four hundred and seventy-eight eligible participants were recruited to the study (intervention group $n=239$; comparison group $n=239$ ) and attended baseline measurement. Baseline characteristics were similar in the two trial groups (Table 1). The majority of ADDITION-Plus participants were white men with a mean age of 60 years. In all, $51 \%$ of participants were in full- or part-time employment, and $62 \%$ had attended full-time education after the age of 16 years. There was one death in the comparison group and two deaths in the intervention group within 13 months of recruitment. Of those still alive, 444/475 (93\%; intervention group: 95\%, comparison group: $92 \%$ ) returned for follow-up health assessment after a mean of 1.1 years (SD 0.2). There was no significant difference in baseline characteristics between those who attended follow-up health assessment and those who did not (data not shown).

\section{Objectively measured health behaviours}

Table 2 shows the outcomes of objectively measured health behaviours. PAEE levels were similar in both groups at 1 year. We observed small increases in plasma vitamin $C$ levels in both groups over 12 months, with levels approximating national trends (National Diet and Nutrition Survey 2002 [39]) and not significantly differing by group. The proportion of smokers was similar in the intervention and comparison groups at 1 year, reflecting national smoking rates [40]. There were no differences between groups in the proportion of patients prescribed metformin, simvastatin and atorvastatin (data not shown), the mean prescribed total daily dose of each drug (data not shown) and the plasma drug levels.

\section{Self-reported health behaviours}

Participants reported increases in levels of total physical activity and consumption of fruit, vegetables and fibre, and reduction in consumption of fat and alcohol over 1 year, with no significant difference between groups (Table 3 ). There was little change in the proportion smoking with no significant

Table 1 Baseline characteristics of ADDITION-Plus trial participants $(n=478)$

\begin{tabular}{lll}
\hline Participant characteristics & $\begin{array}{l}\text { Comparison group } \\
(n=239)\end{array}$ & $\begin{array}{l}\text { Intervention group } \\
(n=239)\end{array}$ \\
\hline Mean age (SD), years & $59.8(7.5)$ & $59.5(7.5)$ \\
Men & $148(61.9)$ & $150(62.8)$ \\
White ethnicity & $234(97.9)$ & $232(97.1)$ \\
$\begin{array}{l}\text { In full- or part-time } \\
\text { employment }\end{array}$ & $122 / 238(51.3)$ & $123 / 239(51.5)$ \\
$\begin{array}{l}\text { Full-time education after } \\
\quad 16 \text { years of age }\end{array}$ & $145 / 236(61.4)$ & $146 / 236(61.9)$ \\
\hline
\end{tabular}

Values are $n(\%)$ unless specified 
Table 2 Objectively measured health behaviours by study group and between-group differences at 1 year in the ADDITION-Plus trial

\begin{tabular}{|c|c|c|c|c|}
\hline Health behaviours & Comparison group & Intervention group & Difference between groups $(95 \% \mathrm{CI})$ & $p$ value \\
\hline PAEE, $\mathrm{kJ} \mathrm{kg}^{-1}$ day $^{-1 \mathrm{a}}$ & $33.7(15.9)(N=212)$ & $35.2(18.2)(N=218)$ & $+1.50(-1.74,4.74)$ & 0.36 \\
\hline Plasma vitamin $C, \mu \mathrm{mol} / 1$ & $56.2(25.1)(N=216)$ & $53.4(25.0)(N=218)$ & $-3.84(-8.07,0.38)^{\mathrm{b}}$ & 0.07 \\
\hline Smoking, $\%$; cotinine validated $(n / N)$ & $13.2(23 / 174)$ & $17.3(34 / 197)$ & $1.37^{\mathrm{c}}(0.77,2.43)$ & 0.28 \\
\hline \multicolumn{5}{|l|}{ Drug plasma level, $\mu \mathrm{mol} / 1$} \\
\hline Metformin & $1,310.0(741.7)(N=82)$ & $1,190.5(725.6)(N=100)$ & $-119.5(-335.0,95.9)$ & 0.28 \\
\hline Log simvastatin ${ }^{\mathrm{d}}$ & $-1.07(1.73)(N=30)$ & $-1.43(1.45)(N=42)$ & $-0.36(-1.10,0.39)$ & 0.35 \\
\hline Log atorvastatin $^{\mathrm{d}}$ & $1.07(1.01)(N=40)$ & $0.91(1.02)(N=32)$ & $-0.16(-0.63,0.32)$ & 0.52 \\
\hline
\end{tabular}

Values are mean SD unless otherwise stated; $N$ is the number with data available

${ }^{a}$ Physical activity is individually calibrated where available $(n=351)$, otherwise group calibrated $(n=79)$

${ }^{\mathrm{b}}$ Difference between groups is adjusted for baseline values

${ }^{\mathrm{c}}$ Results are expressed as ORs

${ }^{\mathrm{d}}$ Results are expressed on the natural log-scale since original variable showed substantial skewness

Table 3 Baseline and 1 year follow-up self-reported behaviours by study group and between-group differences in the ADDITION-Plus trial

\begin{tabular}{|c|c|c|c|c|c|}
\hline \multirow[t]{2}{*}{ Self-reported behaviour } & \multicolumn{2}{|c|}{ Comparison group } & \multicolumn{2}{|c|}{ Intervention group } & \multirow{2}{*}{$\begin{array}{l}\text { Adjusted difference between } \\
\text { groups }(95 \% \mathrm{CI})\end{array}$} \\
\hline & Baseline & 1 year & Baseline & 1 year & \\
\hline \multicolumn{6}{|l|}{ Physical activity } \\
\hline Total physical activity, MET h/week & $78.6(48.0)$ & $80.1(49.5)$ & $90.0(55.1)$ & $95.2(55.7)$ & $+7.51(-0.009,15.0)$ \\
\hline Recreational activity, $\mathrm{h} /$ week & $9.63(9.81)$ & $10.4(10.6)$ & $12.0(13.0)$ & $13.1(12.9)$ & $+1.46(-0.40,3.34)^{\mathrm{a}}$ \\
\hline Vigorous activity, h/week & $1.03(2.41)$ & $1.04(2.81)$ & $1.33(3.59)$ & $1.42(3.39)$ & $+0.23(-0.32,0.72)^{\mathrm{a}}$ \\
\hline Time spent in sedentary activity, $\mathrm{h} /$ week & $38.7(19.6)$ & $36.4(17.7)$ & $35.3(15.5)$ & $33.4(16.3)$ & $-0.60(-2.57,1.38)$ \\
\hline Television viewing, $\mathrm{h} /$ week & $24.4(12.8)$ & $23.9(11.7)$ & $24.0(10.2)$ & $23.4(10.3)$ & $-0.20(-1.49,1.10)$ \\
\hline $\begin{array}{l}\text { Proportion reporting change in physical } \\
\text { activity at } 1 \text { year, } \%\end{array}$ & - & $41.0(91 / 222)$ & - & $74.9(170 / 227)$ & $4.29(2.87,6.42)^{\mathrm{b}}$ \\
\hline \multicolumn{6}{|l|}{ Diet } \\
\hline Total fat, g/day & $68.7(30.3)$ & $60.3(23.3)$ & $68.3(29.6)$ & $60.6(22.2)$ & $+0.41(-3.11,3.94)$ \\
\hline Fat as percentage of energy, $\%$ & $32.0(6.2)$ & $31.2(6.0)$ & $31.3(5.7)$ & $30.4(5.9)$ & $-0.45(-1.41,0.51)$ \\
\hline Polyunsaturated:saturated fat ratio & $0.58(0.23)$ & $0.67(0.27)$ & $0.62(0.28)$ & $0.68(0.26)$ & $-0.01(-0.06,0.03)$ \\
\hline Fibre, g/day & $18.6(7.3)$ & $19.0(7.3)$ & $18.6(7.2)$ & $19.8(7.0)$ & $0.82(-0.31,1.95)$ \\
\hline Plasma vitamin $\mathrm{C}, \mathrm{mg} /$ day & $147.4(71.2)$ & $145.5(66.0)$ & $141.0(72.2)$ & $146.2(66.5)$ & $3.89(-6.62,14.40)$ \\
\hline Total energy, kJ & $7,966(2,628)$ & $7,200(2,092)$ & $8,099(2,757)$ & $7,446(2,110)$ & $188.0(-136.5,512.5)$ \\
\hline Fruit food group (11 items), g/day & 277 (197) & $312(222)$ & $266(198)$ & $312(212)$ & $4.89(-31.2,41.2)^{\mathrm{a}}$ \\
\hline Vegetable food group (19 items), g/day & $237(128)$ & $246(148)$ & $231(161)$ & $247(135)$ & $4.76(-19.0,26.4)^{\mathrm{a}}$ \\
\hline Alcohol, units/week & $8.5(14.3)$ & $7.3(11.1)$ & $9.5(13.6)$ & $7.8(10.6)$ & $-0.25(-1.45,1.24)^{\mathrm{a}}$ \\
\hline Proportion reporting change in diet at 1 year, $\%$ & - & $57.2(127 / 222)$ & - & $77.4(175 / 226)$ & $2.57(1.70,3.87)^{\mathrm{b}}$ \\
\hline \multicolumn{6}{|l|}{ Smoking } \\
\hline Self-reported smokers, $\%$ & $14.0(31 / 222)$ & $11.7(26 / 222)$ & $15.0(34 / 227)$ & $15.0(34 / 227)$ & $2.63(0.76,9.05)^{\mathrm{b}}$ \\
\hline \multicolumn{6}{|l|}{ Medication adherence } \\
\hline Adhering to hypoglycaemic medication, $\%^{\mathrm{c}}$ & - & $81.2(108 / 133)$ & - & $85.7(132 / 154)$ & $1.39(0.74,2.60)^{\mathrm{b}}$ \\
\hline Adhering to general medication, $\%^{\mathrm{c}}$ & $73.1(147 / 201)$ & $75.6(161 / 213)$ & $69.5(148 / 213)$ & $78.4(174 / 222)$ & $1.25(0.79,1.99)^{\mathrm{b}}$ \\
\hline
\end{tabular}

Values are mean (SD) unless stated

${ }^{a}$ Model residuals showed non-normality, which could not be resolved after log-transformation. Therefore, 95\% CIs were calculated using the semiparametric bias corrected and accelerated bootstrap regression method (with 1,999 resamples)

${ }^{\mathrm{b}}$ Results are expressed as ORs

${ }^{\mathrm{c}}$ Adherence defined as a score of $>23 / 25$ on the MARS questionnaire 
between-group differences. Self-reported adherence to general medication increased in both groups from baseline to 1 year follow-up. Medication adherence for both general and diabetes medication was high in both groups at 1 year (defined as a score of $>23 / 25$ on the MARS questionnaire), with no significant difference between groups. A significantly higher number of patients in the intervention than in the comparison group reported that they had made positive changes to their diet and physical activity in the preceding 12 months.

\section{Clinical and biochemical measures}

Table 4 shows the baseline and follow-up anthropometric, clinical and biochemical measures. We observed small reductions in cardiovascular risk factors in both groups over 1 year. The majority of between-group differences favoured the intervention group but none achieved statistical significance.

Functional status, healthy utility, anxiety, well-being, quality of life, treatment satisfaction and relational empathy

Participants in the intervention group reported significantly higher levels of SF-36 physical functioning, SF-36 change in health, health utility (EQ-5D) and satisfaction with diabetes services than those in the comparison group (Table 5). There was no intervention effect on the remaining SF-36 measures, state anxiety, diabetes-specific and general well-being, diabetes-related quality of life, diabetes treatment satisfaction or relational empathy.

Beliefs about behavioural intentions, illness perceptions and habit

Behavioural intentions were higher in the intervention than the comparison group at 1 year, achieving statistical significance for physical activity and medication adherence (Table 6). Illness perceptions, perceived behavioural control and behavioural beliefs were similar in both groups at baseline and 1 year. In addition, there were no differences between groups in the strength of habit and diabetes knowledge levels at 1 year.

In total, $93 \%$ of participants attended the introductory and initial three core-intervention sessions. Intervention group participants reported feeling confident in using the skills they had been taught (mean [SD] score 7.9 [1.7] on a scale of 0 to 10). Self-reported use of skills to increase physical activity and to eat a lower-fat diet was relatively high, ranging from $62 \%$ of participants who reported using prompts or reminders to $88 \%$ who reported setting achievable goals. Fewer

Table 4 Baseline and follow-up anthropometric, clinical and biochemical measures by study group and between-group differences in the ADDITIONPlus trial

\begin{tabular}{|c|c|c|c|c|c|}
\hline \multirow[t]{2}{*}{ Measure } & \multicolumn{2}{|c|}{ Comparison group } & \multicolumn{2}{|c|}{ Intervention group } & \multirow{2}{*}{$\begin{array}{l}\text { Adjusted difference between } \\
\text { groups }(95 \% \mathrm{CI})\end{array}$} \\
\hline & Baseline & 1 year & Baseline & 1 year & \\
\hline BMI, $\mathrm{kg} / \mathrm{m}^{2}$ & $32.8(5.7)$ & $32.3(5.7)$ & $32.7(5.3)$ & $32.1(5.2)$ & $-0.11(-0.44,0.22)$ \\
\hline Waist circumference, $\mathrm{cm}$ & $110.7(15.1)$ & $109.5(15.2)$ & $110.9(12.4)$ & $109.1(11.8)$ & $-0.63(-1.70,0.44)$ \\
\hline Body fat, $\%$ & $42.1(11.9)$ & $41.8(11.2)$ & $42.8(12.3)$ & $42.0(11.8)$ & $-0.30(-1.72,1.12)$ \\
\hline Systolic BP, mmHg & $134.4(19.3)$ & $128.3(17.4)$ & $138.2(19.1)$ & $132.1(17.4)$ & $1.76(-0.92,4.43)$ \\
\hline Diastolic BP, mmHg & $79.1(10.9)$ & $75.1(9.8)$ & $81.6(10.0)$ & $77.7(8.8)$ & $1.43(-0.07,2.92)$ \\
\hline Cardiorespiratory fitness, $\mathrm{ml} / \mathrm{kg} / \mathrm{min}$ & - & $30.9(9.5)$ & - & $30.3(8.6)$ & $-0.61(-2.63,1.42)$ \\
\hline $\mathrm{HbA}_{1 \mathrm{c},} \%$ & $7.01(1.23)$ & $6.67(0.95)$ & $7.23(1.62)$ & $6.66(0.94)$ & $-0.09(-0.25,0.07)$ \\
\hline $\mathrm{HbA}_{1 \mathrm{c}}, \mathrm{mmol} / \mathrm{mol}$ & 53.1 & 49.4 & 55.5 & 49.3 & \\
\hline Total cholesterol, mmol/1 & $4.90(1.16)$ & $4.31(0.86)$ & $4.96(0.98)$ & $4.33(0.91)$ & $-0.005(-0.16,0.15)$ \\
\hline HDL-cholesterol, mmol/1 & $1.20(0.35)$ & $1.19(0.32)$ & $1.17(0.35)$ & $1.19(0.31)$ & $0.02(-0.02,0.05)$ \\
\hline LDL-cholesterol, mmol/1 & $2.87(0.99)$ & $2.34(0.76)$ & $2.89(0.91)$ & $2.29(0.77)$ & $-0.06(-0.19,0.08)$ \\
\hline $\log _{10}$ triacylglycerol, $\mathrm{mmol} / \mathrm{l}$ & $0.23(0.24)$ & $0.21(0.23)$ & $0.28(0.22)$ & $0.24(0.24)$ & $-0.006(-0.04,0.03)$ \\
\hline $\log _{10}$ alanine aminotransferase & $1.61(0.19)$ & $1.53(0.18)$ & $1.63(0.21)$ & $1.53(0.19)$ & $-0.01(-0.04,0.02)$ \\
\hline Microalbuminuria, $\%{ }^{\mathrm{a}}$ & $20.2 \%(43 / 213)$ & $16.1 \%(35 / 217)$ & $18.6 \%(41 / 220)$ & $17.5 \%(39 / 223)$ & $1.22(0.68,2.17)^{\mathrm{b}}$ \\
\hline Rose angina questionnaire, $\%$ positive & - & $12.2 \%(27 / 222)$ & - & $12.0 \%(27 / 225)$ & $0.98(0.56,1.74)^{b}$ \\
\hline Michigan neuropathy score & $1.91(1.67)$ & $1.91(1.88)$ & $1.84(1.61)$ & $1.78(1.71)$ & $-0.08(-0.34,0.18)$ \\
\hline $\log _{10}$ UKPDS 10 year CVD risk ${ }^{\mathrm{c}}$ & $-0.63(0.21)$ & $-0.68(0.21)$ & $-0.62(0.23)$ & $-0.68(0.22)$ & $-0.02(-0.04,0.006)$ \\
\hline
\end{tabular}

Values are mean (SD) unless stated

${ }^{\text {a }}$ Albumin/creatinine ratio $\geq 2.5$ (men), $\geq 3.5$ (women)

${ }^{\mathrm{b}}$ Results expressed as ORs

${ }^{\mathrm{c}}$ Excluding $n=53$ reporting prior CVD 
Table 5 Functional status, health utility, anxiety, quality of life, well-being and satisfaction with treatment at 1 year by study group and between-group differences in the ADDITION-Plus trial

\begin{tabular}{|c|c|c|c|}
\hline Measure $^{\mathrm{a}}$ & Comparison group & Intervention group & Difference between groups $(95 \% \mathrm{CI})^{\mathrm{b}}$ \\
\hline \multicolumn{4}{|l|}{ SF-36 } \\
\hline Physical functioning & $73.3(27.8)$ & $79.2(22.5)$ & $5.89(1.24,10.85)$ \\
\hline Role limitation, physical & $71.7(40.2)$ & $76.0(35.8)$ & $4.32(-3.06,11.29)$ \\
\hline Role limitation, emotional & $84.4(32.2)$ & $80.7(34.2)$ & $-3.69(-9.98,1.99)$ \\
\hline Social functioning & $83.6(25.3)$ & $85.1(23.9)$ & $1.51(-2.93,6.02)$ \\
\hline Mental health & $76.3(19.4)$ & $76.5(17.3)$ & $0.15(-3.25,3.32)$ \\
\hline Energy/vitality & $56.5(24.0)$ & $59.4(20.8)$ & $2.97(-1.11,7.21)$ \\
\hline Pain & $71.6(27.3)$ & $73.1(25.2)$ & $1.50(-3.28,6.37)$ \\
\hline General health perception & $59.7(23.4)$ & $61.2(20.6)$ & $1.46(-2.98,5.51)$ \\
\hline Change in health & $55.4(20.9)$ & $64.1(22.7)$ & $8.72(4.57,12.74)$ \\
\hline \multicolumn{4}{|l|}{ Health utility } \\
\hline Self-reported general health (1 to 5) & $2.94(0.92)$ & $3.11(0.87)$ & $0.19(0.07,0.31)^{\mathrm{c}}$ \\
\hline EQ-5D $(-0.3$ to 1.0$)$ & $0.81(0.26)$ & $0.84(0.20)$ & $0.03(0.003,0.06)^{\mathrm{c}}$ \\
\hline \multicolumn{4}{|l|}{ Anxiety } \\
\hline Spielberger State Anxiety (20 to 80 ) & $30.5(11.0)$ & $29.6(9.6)$ & $-0.85(-2.56,0.85)^{\mathrm{c}}$ \\
\hline \multicolumn{4}{|l|}{ Quality of life and well-being } \\
\hline Quality of life ( -9 to 9$)$ & $0.96(1.28)$ & $0.79(1.05)$ & $-0.17(-0.38,0.06)$ \\
\hline Diabetes-specific well-being ( 0 to 36 ) & $28.2(6.6)$ & $28.8(6.3)$ & $0.61(-0.70,1.76)$ \\
\hline General well-being ( 0 to 36 ) & $26.4(7.2)$ & $26.9(6.5)$ & $0.47(-0.80,1.81)$ \\
\hline \multicolumn{4}{|l|}{ Satisfaction with treatment, services and empathy } \\
\hline Diabetes treatment satisfaction ( 0 to 36$)$ & $30.0(5.8)$ & $30.6(5.3)$ & $0.59(-0.41,1.72)$ \\
\hline General practitioner CARE measure (10 to 50 ) & $39.1(10.2)$ & $40.3(9.5)$ & $1.23(-0.64,3.17)$ \\
\hline Nurse CARE measure (10 to 50 ) & $39.8(9.8)$ & $40.9(9.1)$ & $1.14(-0.78,2.89)$ \\
\hline Satisfaction with diabetes services ( 1 to 4 ) & $3.43(0.79)$ & $3.68(0.56)$ & $0.25(0.13,0.38)$ \\
\hline
\end{tabular}

Values are mean (SD) unless stated

${ }^{\text {a }}$ Values in parentheses represent the possible range for each measure

${ }^{\mathrm{b}}$ Model residuals showed non-normality, which could not be resolved after log-transformation. Therefore, $95 \%$ CIs were calculated using the semiparametric bias corrected and accelerated bootstrap regression method (with 1,999 resamples)

${ }^{\mathrm{c}}$ Difference between groups is adjusted for baseline values

participants reported using these skills to enhance medication adherence, ranging from $50 \%$ who reported that they had prepared for setbacks to $68 \%$ setting achievable goals. Skills use was lowest among those participants who were trying to stop smoking, with $26 \%$ recording or monitoring their progress and $45 \%$ reporting that they had set achievable goals. Intervention participants rated their facilitators highly with a mean response of 44 (SD 6.5, on a scale of 10-50).

Per protocol, subgroup and sensitivity analyses

Per protocol analyses of the primary outcome data replicated our main results, except for the plasma vitamin $\mathrm{C}$ level for which the increase over 12 months was slightly greater in the comparison than in the intervention group $(p=0.04)$. Our conclusions were unaffected by the sensitivity analysis for missing data. Similarly, there was no evidence that intervention effects on the primary outcomes differed between participants with screen-detected and recently diagnosed diabetes.

\section{Discussion}

For patients with recently diagnosed diabetes offered intensive treatment in primary care the additional input of a facilitator from outside the practice delivering a theory-based multibehaviour intervention was not associated with significant improvements in objectively measured health behaviours. There was no difference between trial groups in change from baseline to 1 year in cardiovascular risk factors or self-reported health behaviours. The intervention group reported higher levels of SF-36 physical functioning as well as SF-36 change in health status, health utility and satisfaction with diabetes services at 1 year, and greater changes in diet and activity over the year 
Table 6 Baseline and follow-up behavioural beliefs, illness perceptions, strength of habit and diabetes knowledge by study group and between-group differences in the ADDITION-Plus trial

\begin{tabular}{|c|c|c|c|c|c|}
\hline \multirow[t]{2}{*}{ Measures $^{\mathrm{a}}$} & \multicolumn{2}{|c|}{ Comparison group } & \multicolumn{2}{|c|}{ Intervention group } & \multirow{2}{*}{$\begin{array}{l}\text { Adjusted difference between } \\
\text { groups }(95 \% \mathrm{CI})\end{array}$} \\
\hline & Baseline & 1 year & Baseline & 1 year & \\
\hline Intention physical activity ( 1 to 5 ) & $3.72(0.83)$ & $3.48(0.79)$ & $3.78(0.74)$ & $3.64(0.81)$ & $0.14(0.007,0.27)$ \\
\hline Intention diet ( 1 to 5 ) & $3.71(0.79)$ & $3.39(0.84)$ & $3.75(0.76)$ & $3.51(0.81)$ & $0.11(-0.03,0.25)$ \\
\hline Intention medication adherence ( 1 to 5 ) & $4.45(0.56)$ & $4.47(0.60)$ & $4.40(0.67)$ & $4.57(0.58)$ & $0.12(0.01,0.23)$ \\
\hline Intention smoking ( 1 to 5 ) & $3.16(1.11)$ & $3.12(1.06)$ & $3.20(0.96)$ & $3.20(1.11)$ & $0.003(-0.32,0.33)$ \\
\hline Illness perception consequences ( 1 to 5 ) & $2.90(0.65)$ & $2.88(0.69)$ & $2.89(0.64)$ & $2.95(0.66)$ & $0.08(-0.03,0.18)$ \\
\hline Illness perception treatment control ( 1 to 5 ) & $3.80(0.52)$ & $3.67(0.55)$ & $3.75(0.48)$ & $3.72(0.52)$ & $0.08(-0.01,0.16)$ \\
\hline Perceived behavioural control physical activity ( 1 to 5 ) & $3.72(0.91)$ & $3.51(0.90)$ & $3.82(0.79)$ & $3.67(0.88)$ & $0.12(-0.03,0.26)$ \\
\hline Perceived behavioural control diet ( 1 to 5 ) & $3.75(0.83)$ & $3.58(0.85)$ & $3.70(0.89)$ & $3.67(0.87)$ & $0.11(-0.03,0.26)$ \\
\hline Perceived behavioural control medication ( 1 to 5 ) & $4.49(0.56)$ & $4.47(0.56)$ & $4.47(0.63)$ & $4.56(0.58)$ & $0.10(-0.002,0.20)$ \\
\hline Perceived behavioural control smoking ( 1 to 5 ) & $2.70(0.97)$ & $2.90(0.79)$ & $2.79(1.01)$ & $2.91(1.07)$ & $-0.07(-0.44,0.30)$ \\
\hline Behavioural beliefs physical activity ( 1 to 5 ) & $4.01(0.75)$ & $3.92(0.64)$ & $3.98(0.62)$ & $3.90(0.72)$ & $-0.002(-0.11,0.11)$ \\
\hline Behavioural beliefs diet ( 1 to 5 ) & $3.83(0.75)$ & $3.75(0.75)$ & $3.90(0.68)$ & $3.81(0.73)$ & $0.02(-0.10,0.14)$ \\
\hline Perceived effectiveness of lifestyle change (1 to 5) & $3.95(0.72)$ & $3.82(0.76)$ & $3.91(0.65)$ & $3.84(0.71)$ & $0.05(-0.08,0.18)$ \\
\hline Strength of PA habit change reported at 1 year (1 to 5) & - & $3.27(0.72)$ & - & $3.41(0.71)$ & $0.14(-0.05,0.32)$ \\
\hline Strength of diet habit change reported at 1 year ( 1 to 5 ) & - & $3.66(0.55)$ & - & $3.64(0.61)$ & $-0.02(-0.15,0.12)$ \\
\hline Diabetes knowledge (self-administered questionnaire) (0 to 47) & - & $24.8(9.18)$ & - & $25.8(8.53)$ & $0.92(-0.72,2.57)$ \\
\hline
\end{tabular}

Values are mean (SD) unless stated

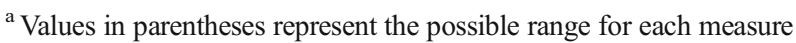

PA, physical activity

compared with the comparison group. However, there was no intervention effect on the remaining SF-36 measures, state anxiety, diabetes-specific and general well-being, diabetesrelated quality of life, diabetes treatment satisfaction or relational empathy. We found no evidence to support this external behavioural facilitator model of care where the primary care team delivers a well-organised and intensive treatment service.

Strengths and limitations

The study was carried out in primary care settings where most of the care of individuals with recently diagnosed diabetes takes place in the UK. General practice registers typically cover $99 \%$ of all residents living in England [41], and nearly half of the practices approached agreed to take part. Generalisability to more deprived settings with greater ethnic diversity may be limited in light of the non-random recruitment of general practices from a single geographical region.

Internal validity was strong; participants were individually randomised and groups were well matched for baseline characteristics. Participant retention at 1 year was similarly high in both trial groups. We used objective measurement of four key behaviours affecting CVD risk, as well as measuring selfreported behaviour, functional status and well-being. The apparent effect on self-reported change in behaviours over time highlights the need for caution in interpreting the results of trials of behavioural interventions that rely on subjective measures. Clinically important outcomes were measured using standardised equipment and protocols, with trained staff unaware of study group allocation.

The intervention was based on theory and evidence from psychology to support change in behaviour [42], and included a patient-centred approach to facilitating behaviour change, which has been shown to be more effective than didactic interventions in improving CVD risk factors [43, 44]. Quality-assured delivery was enabled by training, ongoing supervision and protocols. The carefully characterised intervention and the objective measurement of health behaviours allowed us to isolate potential effects of the behavioural intervention from other aspects of intensive general practice care.

Plasma drug and cotinine levels were available only for a subset of participants and the timing of blood samples following ingestion of tablets and other factors affecting plasma drug levels were not standardised, which will have reduced the precision of estimates. However, these issues did not differ by study group and while the measure of adherence was less precise, it was less prone to bias than self-report measures. Objective measures of physical activity, smoking and medication adherence at baseline would have improved precision and enabled us to assess change over time. However, such detailed measurement might increase the salience and influence the behaviour of participants in both groups [45]. This is 
likely to be more relevant to physical activity than to the other health behaviours assessed via blood samples.

\section{Understanding the results}

There may have been limited scope for additional benefit among ADDITION-Plus patients, who were already receiving intensive treatment, including theory-based educational materials and lifestyle advice on all the target behaviours by the primary care team. In addition, there were improvements from baseline in plasma vitamin $\mathrm{C}$ levels, self-reported diet and physical activity and cardiovascular risk factors in both study groups, further limiting potential change. The absence of an intervention effect on health behaviours was unlikely to be due to failure to deliver the programme. Attendance at intervention sessions, self-reported use of skills to improve lifestyle behaviours and levels of satisfaction with the programme were all high. Facilitators met monthly to discuss intervention delivery and listened to tape recordings of their sessions. They also received ongoing supervision and support from a clinical psychologist. The intervention was associated with stronger intentions to change physical activity and medication adherence at follow-up. We targeted mediators of behaviour change and included behaviour change techniques from a range of theories. However, the evidence that any of the commonly used theories predict changes in objectively assessed health behaviours is limited. Behaviour change might be enhanced by focusing on fewer behaviours and being more directive in selecting which ones to focus on. Targeting dietary change and physical activity sequentially might be less effective than targeting them simultaneously, but more evidence is needed [46]. Increasing the intensity of the training or of intervention delivery could have enhanced the opportunity to change behaviour, but may not be feasible in routine practice. Facilitator-led care focusing on behaviour change might add value in settings where primary care based intensive treatment is not feasible or effective.

We recorded moderate effects of the intervention on measures of self-reported health status, health utility and physical function. These positive effects occurred independently of any change in objectively measured health behaviours, suggesting a mechanism independent of behaviour change. Other trials have also shown that interventions can improve patientreported outcomes such as well-being but not behaviour or clinical endpoints $[33,47]$. It is not clear whether the improvements were related to the supportive alliances formed with lifestyle facilitators or whether the effect was mediated by a feeling of false reassurance from participating in the intervention [48]. Regardless of the mechanism, self-reported health is an independent predictor of mortality [49]. These improvements warrant further examination, particularly if they persist over the long term when intervention support is withdrawn and can be achieved with a less intensive intervention. Fiveyear follow-up of the trial cohort is planned.
Recent attempts to promote behaviour change in individuals soon after the diagnosis of diabetes have had mixed effects. In the Diabetes Education and Self-Management for Ongoing and Newly Diagnosed Diabetes (DESMOND) trial (ISRCT N17844016), 824 newly diagnosed patients were cluster randomised to receive either a $6 \mathrm{~h}$ structured group-education programme focused on behaviour change or usual care (comparison group) [50]. The DESMOND trial participants had a mean age of 60 years, included more men than women (55\%), had a mean BMI of approximately $32 \mathrm{~kg} / \mathrm{m}^{2}$ and were, therefore, broadly similar to ADDITION-Plus participants. The intervention was based on similar theories of learning used in the ADDITION-Plus trial and focused on lifestyle factors such as food choice and physical activity. Compared with the control group, the intervention group achieved greater improvements in weight loss and smoking cessation and positive improvements in belief about illness, but no difference in $\mathrm{HbA}_{1 \mathrm{c}}$ levels after 1 year. Self-reported physical activity levels were higher in the intervention than in the comparison group at all time points, but were not significantly different at 1 year. There was no objective measurement of behaviour change in this trial cohort and it was difficult to elucidate exactly which components of the intervention were associated with the observed improvements. Further, the DESMOND control group was different from our comparison group, where individuals were already receiving intensive treatment for diabetes.

In the Early ACTID trial, 593 recently diagnosed patients with a mean age of 60 years were randomised to receive usual care (control group), an intensive diet intervention ( $6.5 \mathrm{~h}$ of individual counselling by a dietitian/nurse over 1 year) or an intensive diet intervention plus a pedometer-based activity programme [7]. After 12 months, there were significant improvements in glycaemic control, insulin resistance and body weight in both intervention groups compared with the control group; however, the addition of the activity intervention conferred no extra benefit. Accelerometry data indicated that individuals in the diet and activity group increased their physical activity significantly more than those in the other two groups. However, the lack of measurement of dietary change or any other health behaviour again makes it difficult to explain the beneficial effects observed in this trial. Other studies examining behavioural change in type 2 diabetes patients tend to be small, of shorter duration and to focus on individuals later in the disease trajectory. No published trial to date captures objective measurement of four key health behaviours in recently diagnosed patients.

\section{Conclusion}

A facilitator-led, individually tailored multiple behaviour change intervention over 1 year did not improve objectively measured health behaviours or cardiovascular risk factors 
among people with recently diagnosed type 2 diabetes receiving intensive treatment in UK primary care. Effects on selfreported changes in diet and physical activity were not confirmed by objective data, highlighting the need for caution in interpreting trials of behavioural interventions that rely on subjective measures. Enabling primary care teams to deliver a well-organised intensive treatment service to newly diagnosed patients should remain a priority for policymakers and commissioners.

Acknowledgements We are grateful to all participants, lifestyle facilitators, practice nurses and family physicians for taking part in the ADDITION-Plus trial: Arbury Road Surgery, Ashwell Surgery, Birchwood Surgery, Bottisham Medical Practice, Brookfields/Cherry Hinton, Buckden Surgery, Clarkson Surgery, Cornerstone Practice, Cornford House Surgery, Cottenham Surgery, Dr Eaton \& Partners (Saffron Walden), George Clare Surgery, Great Staughton Surgery, Haddenham Surgery, Hilton House Surgery, Lensfield Road Surgery, Manea Surgery, Milton Surgery, New Roysia Surgery, Orchard House Surgery, Orton Medical Practice, Parkhall Road Surgery, Park Medical Centre, Petersfield Medical Practice, Riverside Practice, Rookery Medical Centre, Rosalind Franklin House, South Street Surgery, St Mary's Surgery, Thaxted Surgery, The Old Exchange, The Spinney Surgery, The Surgery (Over) and Woolpit Surgery. We are grateful to the independent endpoint committee (N. Stott [Chair; University of Cardiff, UK], J. Weinman [King's College, London, UK], R. Himsworth [University of Cambridge, UK] and P. Little [University of Southampton, UK]). We thank D. Hughes (University of Bangor, Wales) for his help with examining the medication adherence data. We thank all ADDITION-Plus study team members at The Primary Care Unit, University of Cambridge and the Medical Research Council Epidemiology Unit, Cambridge, UK, who jointly coordinated the baseline and 1 year follow-up phases of the study. We thank the Cambridge University Hospitals NHS Foundation Trust Department of Clinical Biochemistry and the National Institute for Health Research (NIHR) Cambridge Biomedical Research Centre, Core Biochemical Assay Laboratory for carrying out the biochemical assays, and the following groups within the MRC Epidemiology Unit: data management (A. Dickinson), information technology (I. Morrison), technical (M. Sims), physical activity (K. Westgate, S. Mayle) and field epidemiology (P. Roberts, K. Mwanza, J. Grant, F. Whittle and J. Sylvester). Permission to use the Diabetes Treatment Satisfaction Questionnaire was granted by C. Bradley, Health Psychology Research, Royal Holloway University of London, Surrey, UK.

Funding The trial is supported by the Medical Research Council (grant reference no. G0001164), the Wellcome Trust (grant reference no. G061895), National Health Service R\&D support funding (including the Primary Care Research and Diabetes Research Networks) and National Institute of Health Research under its Programme Grants for Applied Research scheme (RP-PG-0606-1259). ALK, KMW \& SJG were members of the NIHR School for Primary Care Research. ALK and SS are NIHR Senior Investigators. The Primary Care Unit is supported by NIHR Research funds. ATP is supported by the NIHR Biomedical Research Centre at Guy's and St Thomas' NHS Foundation Trust and King's College, London, UK. The views expressed in this publication are those of the authors and not necessarily those of the National Health Service, the NIHR, or the UK Department of Health. Bio-Rad provided equipment for $\mathrm{HbA}_{1 \mathrm{c}}$ testing during the screening phase. We are grateful to Diabetes UK for providing patient information materials.

Access to research materials For access to research materials contact the corresponding author.
Duality of interest The authors declare that there is no duality of interest associated with this manuscript.

Author contributions SJG, NJW and ALK were responsible for the conception and design of the study. UE, SJG, WH, ALK, RKS, SS, NJW and KMW were responsible for acquisition of the data. SJG, RAP, ATP, RKS and SB analysed and interpreted the data. RKS and SJG drafted the article and all authors revised it critically for important intellectual content. All authors approved the final version. SJG is responsible for the integrity of the work as a whole.

Open Access This article is distributed under the terms of the Creative Commons Attribution License which permits any use, distribution, and reproduction in any medium, provided the original author(s) and the source are credited.

\section{References}

1. Griffin S (1998) Diabetes care in general practice: meta-analysis of randomised control trials. BMJ 317:390-396

2. Olivarius NF, Beck-Nielsen H, Andreasen AH, Horder M, Pedersen PA (2001) Randomised controlled trial of structured personal care of type 2 diabetes mellitus. BMJ 323:1-9

3. Gaede P, Lund-Andersen H, Parving H-H, Pedersen O (2008) Effect of a multifactorial intervention on mortality in type 2 diabetes. N Engl J Med 358:580-591

4. Ray KK, Seshasai SR, Wijesuriya S et al (2009) Effect of intensive control of glucose on cardiovascular outcomes and death in patients with diabetes mellitus: a meta-analysis of randomised controlled trials. Lancet 373:1765-1772

5. Ellis SE, Speroff T, Dittus RS, Brown A, Pichert JW, Elasy TA (2004) Diabetes patient education: a meta-analysis and metaregression. Patient Educ Couns 52:97-105

6. Department of Health and Diabetes UK (2005) Structured patient education in diabetes - report from the patient education working group. Department of Health, London

7. Andrews RC, Cooper AR, Montgomery AA et al (2011) Diet or diet plus physical activity versus usual care in patients with newly diagnosed type 2 diabetes: the Early ACTID randomised controlled trial. Lancet 378:129-139

8. Wing RR (2010) Long-term effects of a lifestyle intervention on weight and cardiovascular risk factors in individuals with type 2 diabetes mellitus: four-year results of the Look AHEAD trial. Arch Intern Med 170:1566-1575

9. Griffin SJ, Simmons RK, Williams KM et al (2011) Protocol for the ADDITION-Plus study: a randomised controlled trial of an individually-tailored behaviour change intervention among people with recently diagnosed type 2 diabetes under intensive UK general practice care. BMC Public Health 11:211

10. Echouffo-Tcheugui JB, Simmons RK, Williams KM et al (2009) The ADDITION-Cambridge trial protocol-a cluster randomised controlled trial of screening for type 2 diabetes and intensive treatment for screen-detected patients. BMC Public Health 9:136

11. Horne R, Weinman J (1998) Predicting treatment adherence: an overview of theoretical models. In: Myers L, Midence K (eds) Adherence to treatment in medical conditions. Harwood Academic, London, pp 25-50

12. Brage S, Brage N, Franks PW, Ekelund U, Wareham NJ (2005) Reliability and validity of the combined heart rate and movement sensor Actiheart. Eur J Clin Nutr 59:561-570

13. Brage S, Ekelund U, Brage N et al (2007) Hierarchy of individual calibration levels for heart rate and accelerometry to measure physical activity. J Appl Physiol 103:682-692 
14. Tanaka H, Monahan KD, Seals DR (2001) Age-predicted maximal heart rate revisited. J Am Coll Cardiol 37:153-156

15. Stegle O, Fallert SV, MacKay DJ, Brage S (2008) Gaussian process robust regression for noisy heart rate data. IEEE Trans Biomed Eng $55: 2143-2151$

16. Brage S, Brage N, Franks PW et al (2004) Branched equation modeling of simultaneous accelerometry and heart rate monitoring improves estimate of directly measured physical activity energy expenditure. J Appl Physiol 96:343-351

17. Khaw KT, Wareham N, Bingham S, Luben R, Welch A, Day N (2004) Association of hemoglobin A1c with cardiovascular disease and mortality in adults: the European prospective investigation into cancer in Norfolk. Ann Intern Med 141:413-420

18. West R, Hajek P, Foulds J, Nilsson F, May S, Meadows A (2000) A comparison of the abuse liability and dependence potential of nicotine patch, gum, spray and inhaler. Psychopharmacology 149:198-202

19. Wareham NJ, Rennie KL (1998) The assessment of physical activity in individuals and populations: why try to be more precise about how physical activity is assessed? Int J Obes Relat Metab Disord 22(Suppl 2):S30-S38

20. Bingham SA, Gill C, Welch A et al (1997) Validation of dietary assessment methods in the UK arm of EPIC using weighed records, and 24-hour urinary nitrogen and potassium and serum vitamin $\mathrm{C}$ and carotenoids as biomarkers. Int $\mathrm{J}$ Epidemiol 26(Suppl 1):S137-S151

21. Coleman RL, Stevens RJ, Holman R (2012) Updated UKPDS risk engine that estimates primary and secondary cardiovascular disease risk in people with recently-diagnosed or established type 2 diabetes. In: American Diabetes Association (ADA) Conference, Philadelphia, USA

22. Rose GA (1962) The diagnosis of ischaemic heart pain and intermittent claudication in field surveys. Bull World Health Organ 27:645658

23. Feldman EL, Stevens MJ, Thomas PK, Brown MB, Canal N, Greene DA (1994) A practical two-step quantitative clinical and electrophysiological assessment for the diagnosis and staging of diabetic neuropathy. Diabetes Care 17:1281-1289

24. Ware J, Snow KK, Kosinski M, Gandek B (1993) SF-36 health survey. Manual \& interpretation guide. Nimrod Press, Boston, Massachusetts

25. Kind P, Dolan P, Gudex C, Williams A (1998) Variations in population health status: results from a United Kingdom national questionnaire survey. BMJ 316:736-741

26. Marteau TM, Bekker H (1992) The development of a six-item shortform of the state scale of the Spielberger State-Trait Anxiety Inventory (STAI). Br J Clin Psychol 31(Pt 3):301-306

27. Bradley C (1994) Handbook of psychology and diabetes: a guide to psychological measurement in diabetes research and practice. Harwood Academic Publishers, Chur, Switzerland

28. Mercer SW, Maxwell M, Heaney D, Watt GC (2004) The consultation and relational empathy (CARE) measure: development and preliminary validation and reliability of an empathy-based consultation process measure. Fam Pract 21:699-705

29. Ajzen I (1991) The theory of planned behavior. Organ Behav Hum Decis Process 50:179-211

30. Conner M, Sparks P (2005) Theory of planned behaviour and health behaviour. In: Conner M, Norman, P (eds) Predicting health behaviour. Open University Press, Maidenhead, pp 171-222

31. Leventhal L, Benyamino Y, Brownlee S, Diefenbach M, Leventhal EL, Patrick-Miller L, Robitaille C (1997) Illness representations: theoretical foundations. In: Petrie KJ, Weinman J (eds) Perceptions of health and illness. Harwood Academic Publisher, Amsterdam, pp 19-45
32. Moss-Morris R, Weinman J, Petrie KJ, Horne R, Cameron LD, Buick D (2002) The revised illness perception questionnaire (IPQ-R). Psychol Health 17:1-16

33. Kinmonth AL, Woodcock A, Griffin S, Spiegal N, Campbell MJ (1998) Randomised controlled trial of patient centred care of diabetes in general practice: impact on current wellbeing and future disease risk. The Diabetes Care From Diagnosis Research Team. BMJ 317: 1202-1208

34. Verplanken B, Orbell S (2003) Reflections on past behavior: a selfreport index of habit strength. J Appl Pscyhol 33:1313-1330

35. Simmons RK, Griffin SJ, Steele R, Wareham NJ, Ekelund U (2008) Increasing overall physical activity and aerobic fitness is associated with improvements in metabolic risk: cohort analysis of the ProActive trial. Diabetologia 51:787-794

36. Bingham SA, Welch AA, McTaggart A et al (2001) Nutritional methods in the European Prospective Investigation of Cancer in Norfolk. Public Health Nutr 4:847-858

37. White IR, Thompson SG (2005) Adjusting for partially missing baseline measurements in randomized trials. Stat Med 24:993-1007

38. Schafer JL (1999) Multiple imputation: a primer. Stat Methods Med Res 8:3-15

39. Henderson L, Gregory J, Swan G (2002) National diet and nutrition survey: adults aged 19 to 64 years. Types and quantities of foods consumed. HM Stationery Office, Norwich, UK

40. Office of National Statistics (2009) General lifestyle survey. Available from http://data.gov.uk/dataset/general_lifestyle_survey, accessed 21 March 2014

41. Boreham R, Airey C, Erens R, Tobin, R for the Department of Health (2002) National Surveys of NHS patients: general practice, 2002. Available from http://webarchive.nationalarchives.gov.uk/ 20130107105354/http://www.dh.gov.uk/en/Publicationsandstatistics/ Publications/PublicationsStatistics/DH_4119522, accessed 21 March 2014

42. Michie S, Abraham C, Whittington C, McAteer J, Gupta S (2009) Effective techniques in healthy eating and physical activity interventions: a meta-regression. Health Psychol 28:690-701

43. Goldstein MG, Whitlock EP, DePue J (2004) Multiple behavioral risk factor interventions in primary care. Summary of research evidence. Am J Prev Med 27:61-79

44. Norris SL, Zhang X, Avenell A et al (2004) Long-term effectiveness of lifestyle and behavioral weight loss interventions in adults with type 2 diabetes: a meta-analysis. Am J Med 117:762-774

45. van Sluijs EM, van Poppel MN, Twisk JW, van Mechelen W (2006) Physical activity measurements affected participants' behavior in a randomized controlled trial. J Clin Epidemiol 59:404-411

46. King AC, Castro CM, Buman MP, Hekler EB, Urizar GG Jr, Ahn DK (2013) Behavioral impacts of sequentially versus simultaneously delivered dietary plus physical activity interventions: the CALM trial. Ann Behav Med 46:157-168

47. Kinmonth A-L, Wareham NJ, Hardeman W et al (2008) Efficacy of a theory-based behavioural intervention to increase physical activity in an at-risk group in primary care (ProActive UK): a randomised trial. Lancet 371:41-48

48. Marteau TM, Kinmonth AL, Thompson S, Pyke S (1996) The psychological impact of cardiovascular screening and intervention in primary care: a problem of false reassurance? British Family Heart Study Group. Br J Gen Pract 46:577-582

49. Idler EL, Benyamini Y (1997) Self-rated health and mortality: a review of twenty-seven community studies. J Health Soc Behav 38:21-37

50. Davies MJ, Heller S, Skinner TC et al (2008) Effectiveness of the diabetes education and self management for ongoing and newly diagnosed (DESMOND) programme for people with newly diagnosed type 2 diabetes: cluster randomised controlled trial. BMJ 336: $491-495$ 